\title{
Nuclear TEAD4 with SIXI Overexpression is an Independent Prognostic Marker in the Stage I-III Colorectal Cancer
}

This article was published in the following Dove Press journal: Cancer Management and Research

\author{
Tong $\mathrm{Yu}^{\mathrm{l}, 2, *}$ \\ Jinglue Song ${ }^{1,2, *}$ \\ Hui Zhou ${ }^{1,2}$ \\ Tingyu $\mathrm{Wu}^{\mathrm{l}, 2}$ \\ Zhonglin Liang ${ }^{1,2}$ \\ Peng Du ${ }^{1,2}$ \\ Chen-Ying Liu ${ }^{1,2}$ \\ Guanghui Wang ${ }^{3}$ \\ Long Cui iD ${ }^{1,2}$ \\ Yun Liu ${ }^{l, 2}$ \\ 'Department of Colorectal Surgery, \\ Xinhua Hospital, Shanghai Jiao Tong \\ University School of Medicine, Shanghai, \\ People's Republic of China; ${ }^{2}$ Shanghai \\ Colorectal Cancer Research Center, \\ Shanghai, People's Republic of China; \\ ${ }^{3}$ Guizhou Provincial People's Hospital, \\ Guizhou, People's Republic of China \\ *These authors contributed equally to \\ this work
}

\begin{abstract}
Introduction: Stage I-III colorectal cancer patients are under risk of tumor recurrence and metachronous metastasis after radical surgery. An increased expression of transcription factor TEAD4 is associated with epithelial-mesenchymal transition, metastasis and poor prognosis in colorectal cancer. However, the mechanistic role of TEAD4 in driving colon cancer progression and its prognostic value in early stage of CRC remains unclear.

Methods: In this study, the regulation, function and prognostic significance of TEAD4 and its new direct target gene SIX1 in CRC progression were evaluated using human tissues, molecular and cell biology.
\end{abstract}

Results: We show that TEAD4 directly upregulates the expression of SIX1 at transcriptional level in CRC cells, establishing that SIX1 is a new direct target gene of TEAD4. TEAD4 promotes EMT and cell migration of CRC cells, while SIX1 knockdown attenuates this effect and SIX1 overexpression enhances this effect, indicating that SIX1 mediates the function of TEAD4 in promoting cell migration in CRC cells. Clinically, nuclear TEAD4, overexpression of SIX1 and nuclear TEAD4 with SIX1 overexpression predict poor prognosis in CRC patients.

Discussion: Our study identifies TEAD4-SIX1-CDH1 form a novel signaling axis, which contributes to CRC progression, and its aberrant expression and activation predicts poor prognostic for CRC patients in stage I-III.

Keywords: colorectal cancer, TEAD4, SIX1, hippo pathway

\section{Introduction}

Colorectal cancer is the third most common cancer in the world. Although radical surgery is an effective treatment in the stage I-III CRC, some patients are still at high risk of metastasis and with poor prognosis. $14.5 \%$ of stage I-III patients suffered metachronous liver metastasis in 5 years after radical surgery, ${ }^{1}$ thus it is crucial to identify the biomarkers for stratifying patients into different risk group to direct the clinical treatment after radical surgery.

Epithelial-mesenchymal transition (EMT) significantly contributes to the tumor progression, metastasis and recurrence. ${ }^{2}$ Expression of the EMT-related signature in tumors is correlated with poor prognosis, and EMT-related transcription factors thus serve as prognostic markers for CRC patients. ${ }^{3}$ SIX1 is a transcription factor belongs to the sine oculis homeobox family, which plays a vital role in the development of muscle, kidney and the auditory system in the physiological condition. ${ }^{4}$ The role of SIX1 in tumorigenesis has been widely studied in multiple
Correspondence: Yun Liu

Tel + 86-02I-25078825

Email liuyun@xinhuamed.com.cn

Guanghui Wang

Email wangguanghui0625@163.com
Cancer Management and Research 2021:13 158|-1589

$|58|$

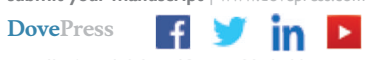

(c) (i) (5) 2021 Yu et al. This work is published and licensed by Dove Medical Press Limited. The full terms of this license are available at https://www.dovepress.com/terms.php cc) you hereby accept the Terms. Non-commercial uses of the work are permitted without any further permission from Dove Medical Press
permission for commercial use of this work, please see paragraphs 4.2 and 5 of our Terms (https://www.dovepress.com/terms php). 
cancers, including colorectal cancer. Overexpression of SIX1 can promote EMT in colorectal cancer cells ${ }^{5}$ and is an independent prognostic marker in colorectal cancer. ${ }^{6}$ However, the mechanism of SIX1 overexpression in CRC is still unknown.

Previously, we reported that TEAD4, one of the downstream transcription factors of Hippo pathway, is overexpressed in the colorectal cancer and nuclear expression of TEAD4 is correlated with EMT, distant metastasis and poor prognosis. ${ }^{7}$ However, it is unknown whether there is the prognostic value of TEAD4 in the early stage of colorectal cancer. Besides, Vimentin was identified as a direct target gene of TEAD4 in CRC, but how TEAD4 affected EMT transcriptional program in CRC cells still need to be explored. Here, we showed that SIX1 is a direct target gene regulated by TEAD4 in colorectal cancer, mediating the function of TEAD4 in inhibiting CDH1 expression and promoting cell migration. In colorectal cancer tissues, nuclear TEAD4 is associated with overexpression of SIX1. Combination of TEAD4 and SIX1 could serve as an independent prognostic marker for stage I-III CRC patients.

\section{Materials and Methods}

\section{Colorectal Cancer Specimen Collection}

All the human colorectal cancer and paired normal mucosa samples for tissue arrays were collected in Department of Colorectal Surgery, Xinhua Hospital, Shanghai Jiao Tong University School of Medicine, from January 2008 to December 2012. Institutional review board approval and informed consent were obtained for all the collections. Three hundred and sixty-two I-III stage CRC specimens were used to prepare tissue arrays and analyzed by immunohistochemistry.

\section{Immunohistochemistry}

The paraffin sections were deparaffinized, rehydrated, and treated according to standard protocol. After incubating with the anti-TEAD4 antibody (1:100; Abcam, ab58310) and antiSIX1 antibody (1:100; Proteintech, 10,709-1-AP) overnight, tissue array slides were washed 3 times with PBS and incubated with HRP-conjugated secondary antibody (GK500710; Gene Company Ltd., Shanghai, China) for $30 \mathrm{~min}$ at room temperature. Following three rinses in PBS, staining was developed with 3,3'-diaminobenzidine (DAB) solution for 10 $\min$. The sections were then counterstained with $0.1 \%$ hematoxylin and sealed with coverslips. Immunohistochemical staining was evaluated at $200 \mathrm{X}$ magnifications with a light microscope (Carl Zeiss, Göttingen, Germany).

\section{Cell Culture and Stable Cell Lines Construction}

All cell lines were purchased commercially from ATCC. Colorectal cancer cell lines LoVo and HCT116 were cultured in Dulbecco's modified Eagle media (DMEM) supplemented with $10 \%$ fetal bovine serum and penicillin/ streptomycin (100 unit $/ \mathrm{mL} / 100 \mu \mathrm{g} / \mathrm{mL})$ at $37^{\circ} \mathrm{C}$ in a $5 \%$ $\mathrm{CO}_{2}$ atmosphere. Cells were infected with retrovirus (pQCXIH for overexpression) or lentivirus (pLKO.1 for knockdown), and selected for one week in puromycin (pLKO.1) or hygromycin (pQCXIH) to generate stable cell lines. TEAD4 shRNA sequences were described in the previous study. ${ }^{7}$ SIX1 shRNA sequence were as followed: SIX1-shRNA1: 5'-AGCTTGTTTCTGGAGTTG TTT-3'.

\section{Western Blotting}

Cells were harvested and lysed with 1\% NP-40 lysis buffer (50mM Tris- $\mathrm{HCl}, 150 \mathrm{mM} \mathrm{NaCl}, 1 \% \mathrm{NP}-40,1 \mathrm{mM}$ PMSF, $50 \mathrm{mM} \mathrm{NaF}, 1 \mathrm{mM} \mathrm{Na} \mathrm{VO}_{4}$ and $1 \mathrm{X}$ protease inhibitor cocktails (Sigma, P8340)). Proteins were separated in $10 \%$ SDS-PAGE gel and transferred onto nitrocellulose membrane. The membrane was blocked with $5 \%$ skim milk (BD), incubated with primary antibodies (1:1000) in $5 \%$ skim milk at $4{ }^{\circ} \mathrm{C}$ overnight, washed and incubated with HRP-conjugated secondary antibodies (Beyotime, $1: 2000)$ in $5 \%$ skim milk for $1 \mathrm{~h}$ at RT. The membrane was visualized by enhanced chemiluminescence reagents (Millipore). The following antibodies were used for Western blot: TEAD4 (1:1000; Abcam, ab58310), SIX1 (1:1000; Proteintech, 10,709-1-AP), E-cadherin (BD, 610,181), $\beta$-Actin (Sigma, A2228).

\section{RNA Isolation, Quantitative Real Time-PCR, Transwell Assay, Wound Healing Assay, Luciferase Assay and Chromosome Immunoprecipitation}

All experiments were performed as previously described. ${ }^{7}$ The primer sequences used in this study are included in the Supplementary Table 3.

\section{Statistics}

$\chi^{2}$ test was used to compare the SIX1 expression with the clinicopathological feathers and TEAD4 expression in the 
CRC. The Spearman rank coefficient was used as a statistical measure of correlation between SIX1 RNA expression and TEAD4 RNA expression. The KaplanMeier method was used to estimate overall survival for univariate analysis. Multivariate analysis by Cox proportional hazards regression model was performed to assess the effect of SIX1 expression, TEAD4 nuclear expression and combination of SIX1 and TEAD4 in addition to the age, gender, AJCC stages (I-III), and grade of differentiation. For each comparison, we used a students' $t$-test to determine the statistical significance. The results are shown as the mean \pm s.d. All P-values corresponded to two-sided tests, and P-values $<0.05$ were considered to be statistically significant.

\section{Results}

\section{SIXI is a Direct Transcriptional Target of TEAD4 in Colorectal Cancer}

SIX1 was one of the most differentially expressed genes in our microarray analysis of TEAD4 knockdown LoVo cells $^{7}$ and down-regulation of SIX1 in TEAD4 knockdown cells was further confirmed at mRNA level and protein level (Figure 1A) which was correlated with $\mathrm{CDH} 1$ protein expression level. Intriguingly, similar to the Vimentin, both TEAD4 WT and Y429H mutant (a mutation variant of TEAD4 that impairs the interaction between TEAD4 and its coactivators $\mathrm{YAP} / \mathrm{TAZ})^{7}$ rescue expression in the sh1 (which targets the 3-UTR region of TEAD4 gene) TEAD4 knockdown LoVo cells retained the expression of SIX1 and decreased CDH1 expression (Figure 1B), indicating the Hippo pathway nuclear effector YAP may also be dispensable for the SIX1 expression in colorectal cancer.

Two classical MCAT motifs were identified in the promoter of human SIX1 and luciferase reporter of SIX1 and MCAT motifs mutant one were generated for luciferase reporter assay (Figure 1C). Both MCAT motifs were vital for the SIX1 promoter activity; mutation of the two MCAT motifs in the SIX1 promoter significantly impaired the relative luciferase activity (Figure 1D and E). ChIP analysis was further performed to study the direct binding of endogenous TEAD4 in the native SIX1 promoter. We found a robust enrichment of endogenous TEAD4 around the two MCAT motifs in the SIX1 promoter, and knockdown TEAD4 reduced the TEAD4 enrichment in the SIX1 promoter compared to the shRNA control cells (Figure 1F). All these data indicate SIX1 is a direct target gene of TEAD4 in colorectal cancer, independent on the YAP activity.

\section{SIXI Mediates the Function of TEAD4 in Promoting Cell Migration in CRC Cells}

Knockdown of TEAD4 impaired the cell migration of CRC cells. Re-expression of SIX1 in TEAD4 knockdown cells reduced the CDH1 expression level (Figure 2A). Consistently, re-expression of SIX1 also rescued cell motility of TEAD4 knockdown CRC cells by using transwell assay and wound healing assay (Figure 2B and C). Overexpression of both TEAD4 WT and Y429H enhanced SIX1 expression while decreased the CDH1 expression in the less invasive HCT-116 CRC cell line (Figure 3A). Consistently, knockdown of SIX1 in the TEAD4 overexpressed cells retained CDH1 expression (Figure 3B) and abolished the cell migration induced by overexpression of TEAD4 (Figure 3C and D). These data demonstrate that SIX1 is a direct target gene mediating the function of TEAD4 through regulating $\mathrm{CDH} 1$ expression and cell mobility in CRC cells.

\section{Clinical Correlation and the Prognostic Role of TEAD4-Induced SIXI Expression in the Clinic}

Next, we explored the correlation between TEAD4 and SIX1 in the clinical CRC tissues. In 30 CRC samples, SIX1 mRNA levels were correlated with TEAD4 expression level, but not YAP (Figure 4A). Immunohistochemical analysis of CRC tissue arrays with 362 stage I-III CRC tissues further showed that nuclear overexpression of TEAD4 was associated with SIX1 overexpression in $\mathrm{CRC}$ tissues $(\mathrm{P}<0.001)$ (Figure $4 \mathrm{~B}$ and $\mathrm{C}$ ). The expression of SIX1 in tumor is significantly higher than paired normal tissues (Figure 4B and D). No correlation was observed between overexpression of SIX1 and clinicopathological features in our cohort. However, both overexpression of SIX1 (Figure 4E) and nuclear expression of TEAD4 (Figure 4F) were associated with poor overall survival in stage I-III CRC patients. Interestingly, co-expression of SIX1 and nuclear TEAD4 was correlated with the worst overall survival in the stage I-III CRC patients (Figure 4G). Consistent with a previous study, SIX1 expression was an independent prognostic marker for CRC in our cohort (Supplementary Table 1). Multivariate analysis also indicated that both TEAD4 nuclear expression alone (Supplementary Table 2) and 
A
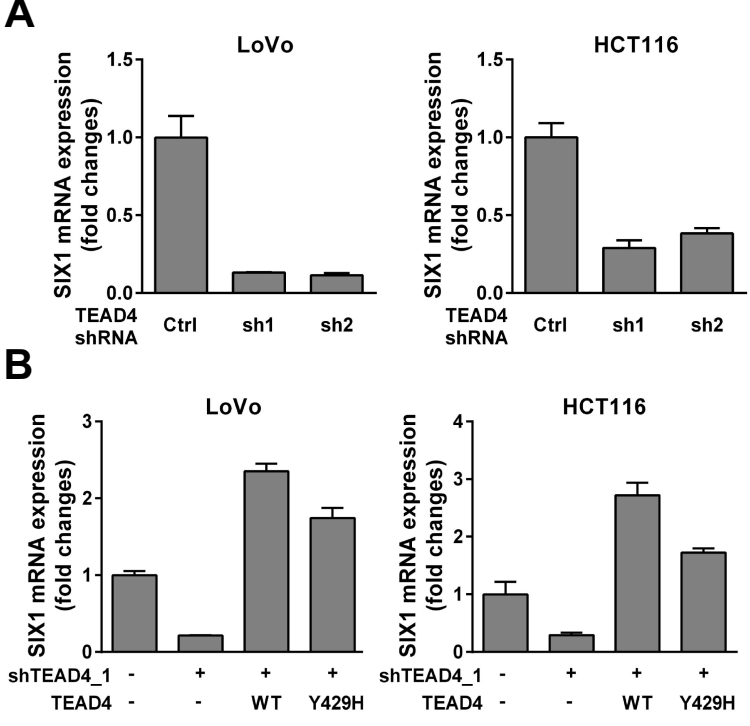

C

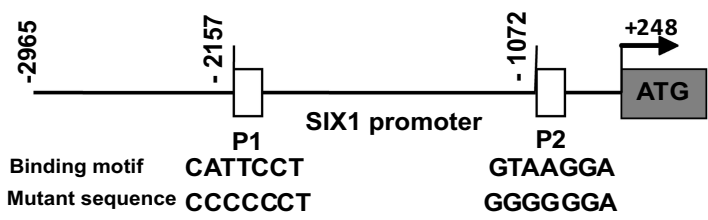

D

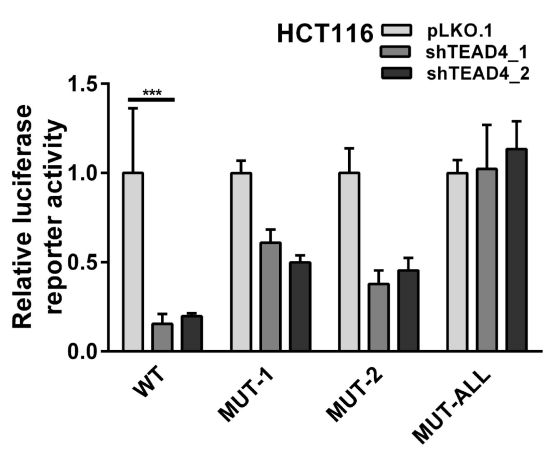

F

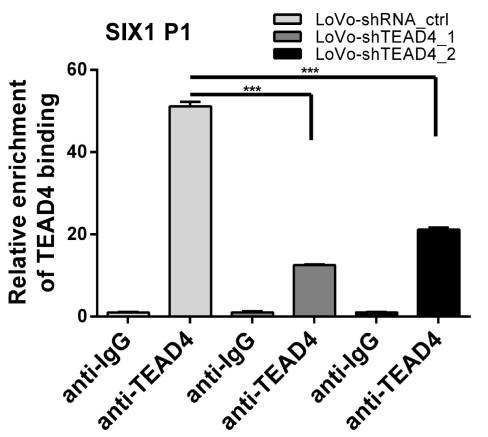

E
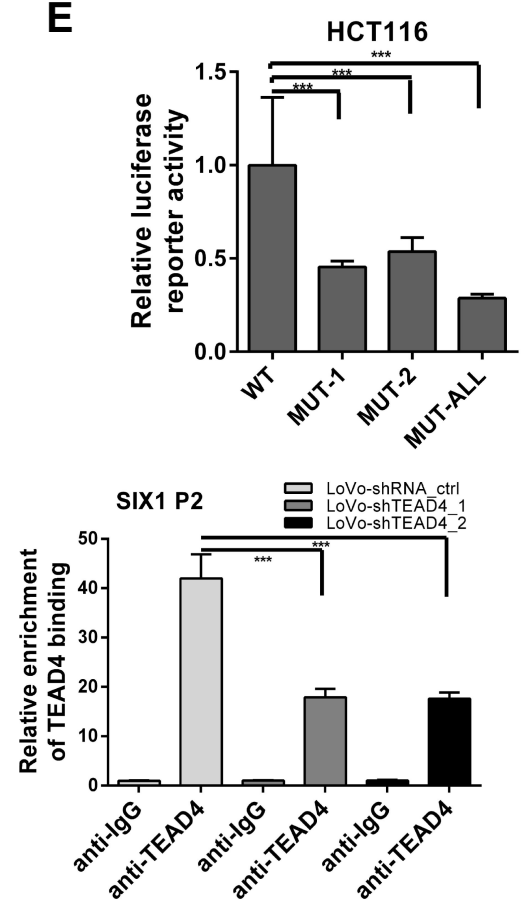

Figure I SIXI is a direct transcriptional target of TEAD4 mediating TEAD4's function of promoting EMT in the colorectal cancer cells. (A) qPCR analysis of TEAD4 expression level in the LoVo cells transduced with control pLKO.I or TEAD4 shRNA virus. Western blot analysis of TEAD4 and SIXI in the control pLKO.I and TEAD4

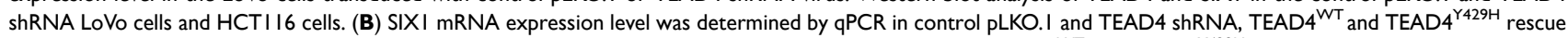
expression LoVo cells. Western blot analysis of TEAD4 and SIXI in control pLKO.I and TEAD4 shRNA, TEAD4 ${ }^{\text {WT }}$ and TEAD4 ${ }^{\text {Y429H }}$ rescue expression LoVo cells. (C) Schematic depiction of the Vimentin promoter with two putative-TEAD4 binding sites (PI and P2) and respective mutant ones as indicated. (D) Relative expression of WT and MCAT binding motif mutant (MUT-I, MUT2, MUT-ALL) SIXI promoter-driven luciferase reporters in control shRNA or TEAD4 shRNA expressing HCTII6 cells. $* * * P<0.00$ I by Student's $t$-test. (E) Relative expression of WT and MCAT binding motif mutant (MUT-I, MUT2, MUT-ALL) SIXI promoter-driven luciferase reporters in control shRNA expressing HCTII 6 cells. $* * * P<0.00$ I by Student's $t$-test. (F) ChIP analysis of TEAD4 binding to the SIXI promoter in the LoVo cells. $q P C R$ was performed with primer specific to regions round the two MCAT motifs (PI and P2). ${ }^{* * * P}<0.00 \mathrm{I}$ by Student's $t$-test. 


\section{A}

B
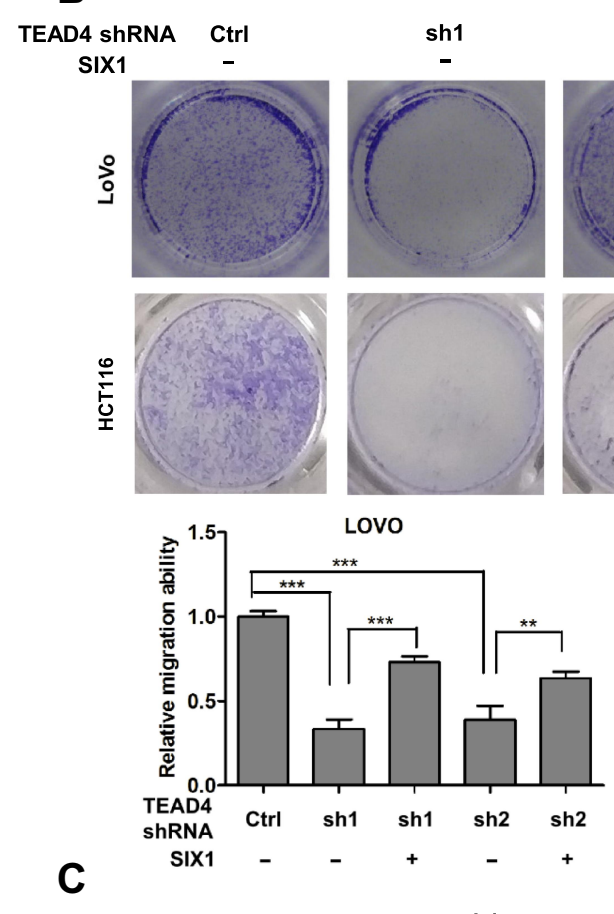

sh1

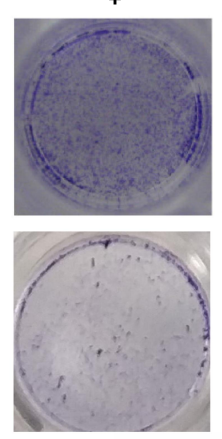

$\geq^{1.5}$
HCT116 cells

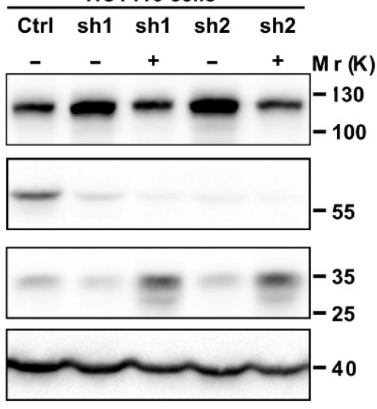

sh2
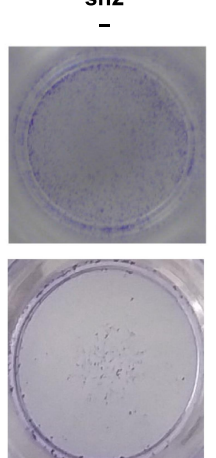

HCT116 sh2

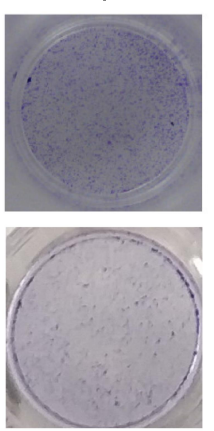

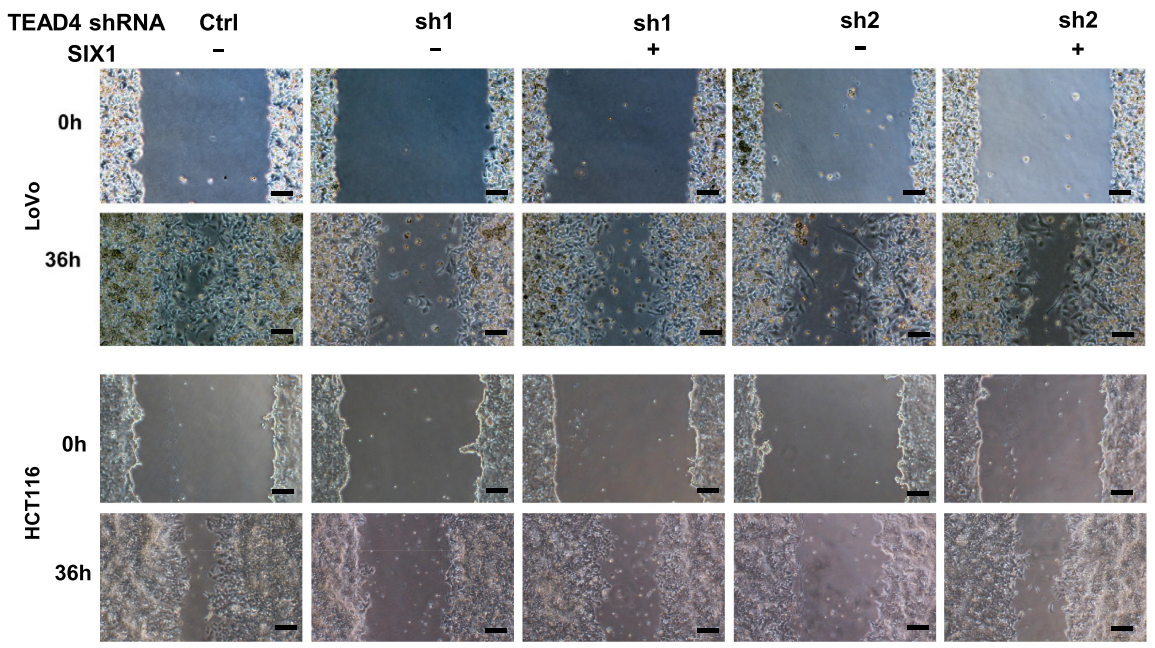

Figure 2 SIXI is required for the TEAD4 function in CRC cells. (A) CDHI, TEAD4, SIXI and $\beta$-ACTIN protein level in each stable cell lines were analyzed by Western blot. (B) Transwell migration assay of each stable LoVo and HCTII6 cell lines. Representative images of total view of the transwell was shown. Data was collected from 10 fields of 3 independent transwell. Relative migration ability was normalized by the control $p L K O$. I group. Student's $t$-tests were performed to assess statistical significance. $* P<0.05$, $* * P<0.01$, $* * * P<0.00 I$. (C) The cell migration of each stable LoVo and HCTII 6 cell lines were determined by wound-healing assay. Monolayer cells were wounded by a sterile plastic pipette tip. Cell migration was observed $36 \mathrm{~h}$ later by microscopy. 
A

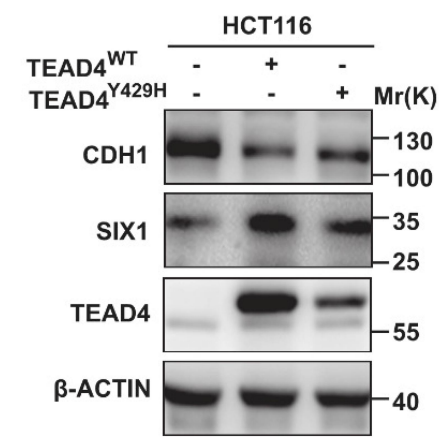

B

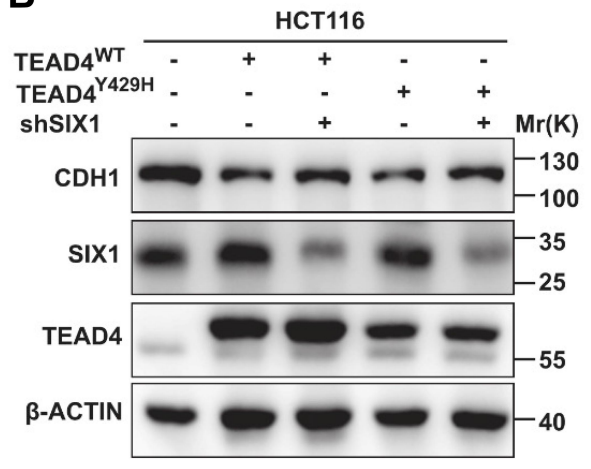

C

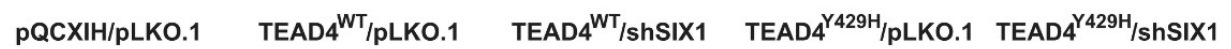

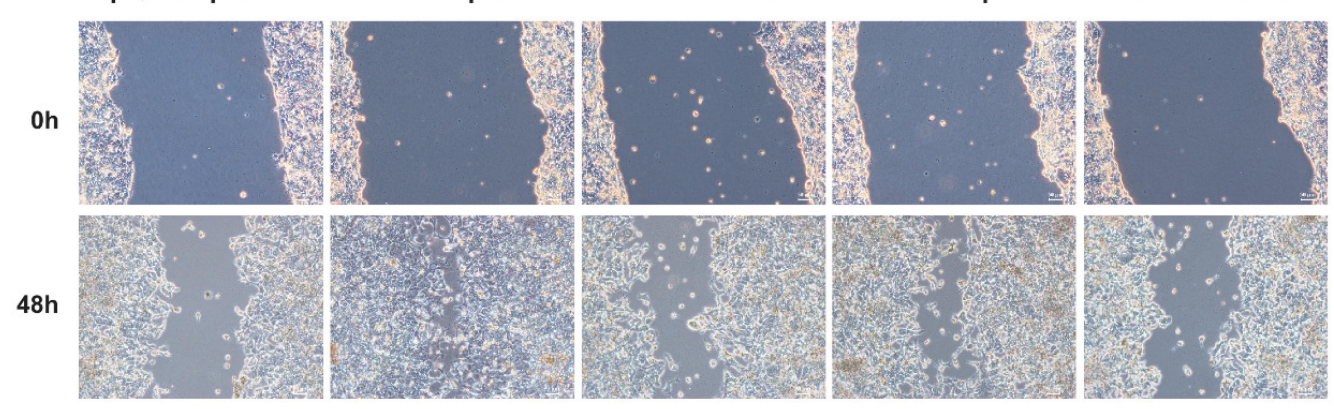

HCT116

D

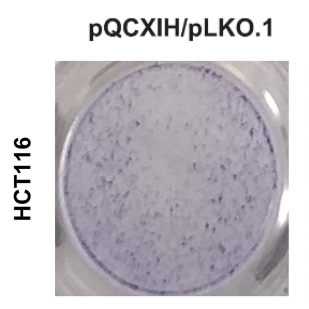

TEAD4 ${ }^{\mathrm{WT}} / \mathrm{pLKO} .1$

TEAD4 ${ }^{\mathrm{WT}} / \mathrm{shSIX} 1$
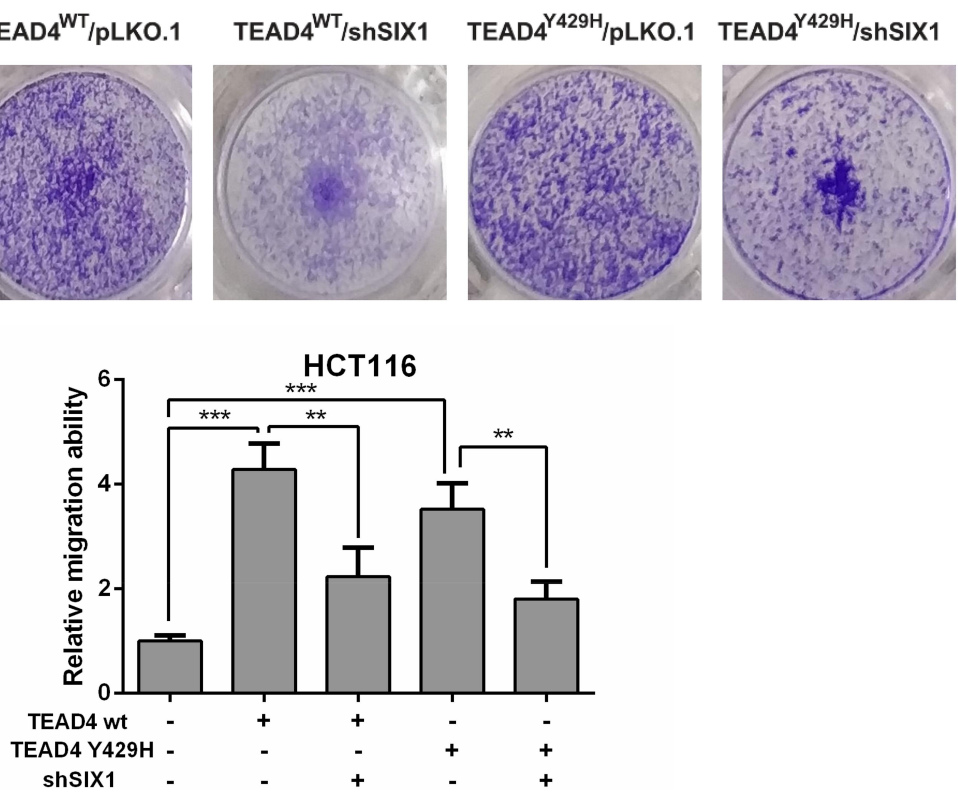

Figure 3 Knockdown SIXI retained the function of TEAD4 in promoting cell migration in CRC cells. (A) Both overexpression of TEAD4 WT and Y429H mutant upregulated the SIXI expression and inhibited CDHI expression in HCTII6 cells. CDHI, TEAD4, SIXI and $\beta$-ACTIN protein level in each stable cell lines were analyzed by Western blot. (B) Knockdown SIXI in the TEAD4 overexpressed HCTII6 cells retained the CDHI expression. CDHI, TEAD4, SIXI and $\beta$-ACTIN protein level in each stable cell lines were analyzed by Western blot. (C) The cell migration of each stable HCTII6 cell lines were determined by wound-healing assay. Monolayer cells were wounded by a sterile plastic pipette tip. Cell migration was observed 36h later by microscopy. (D) Transwell migration assay of each stable HCTI I6 cell lines. Representative images of total view of the transwell was shown. Data was collected from 10 fields of 3 independent transwell. Relative migration ability was normalized by the control PQCXIH/pLKO.I group. Student's $t$-tests were performed to assess statistical significance. $* * P<0.01, * * * P<0.001$. 
A
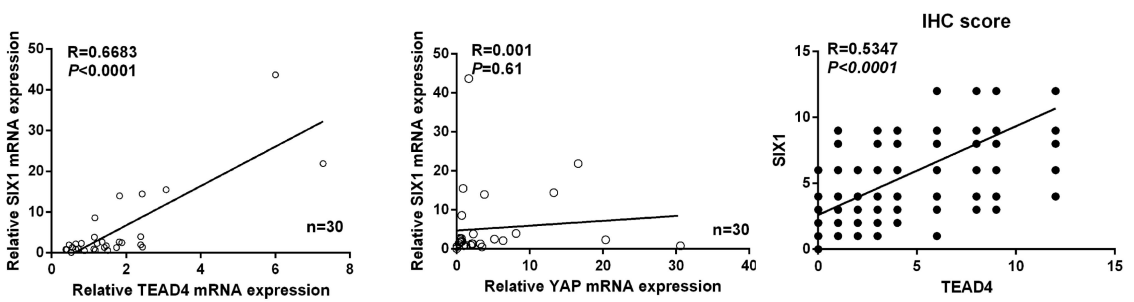

B

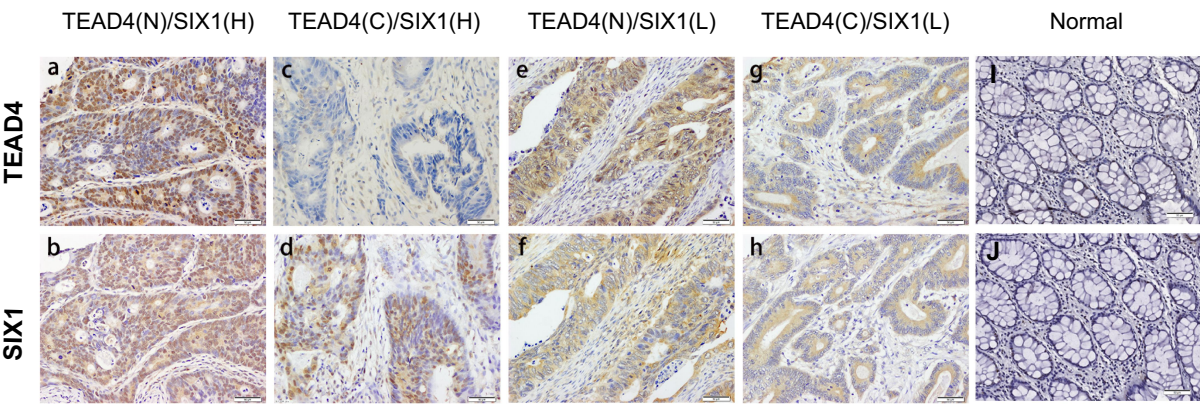

C
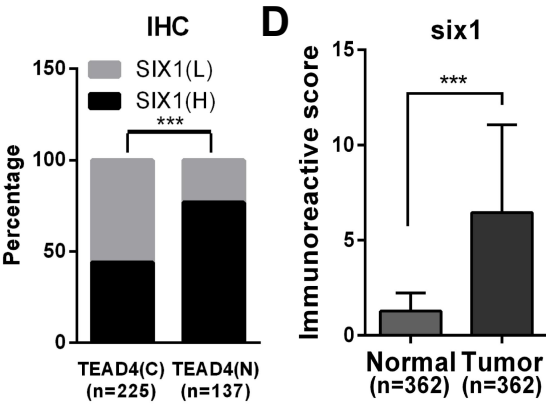

F

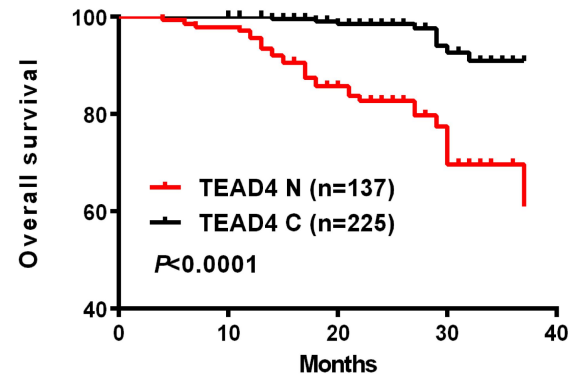

E

SIX1

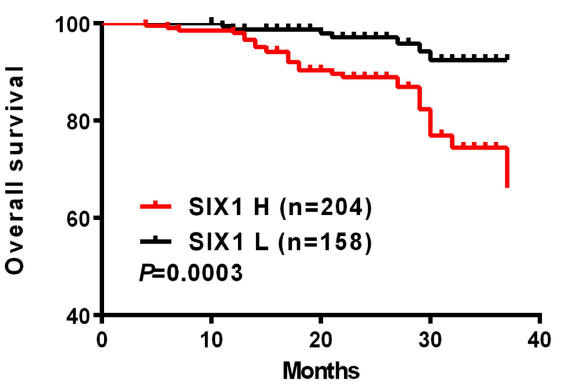

G

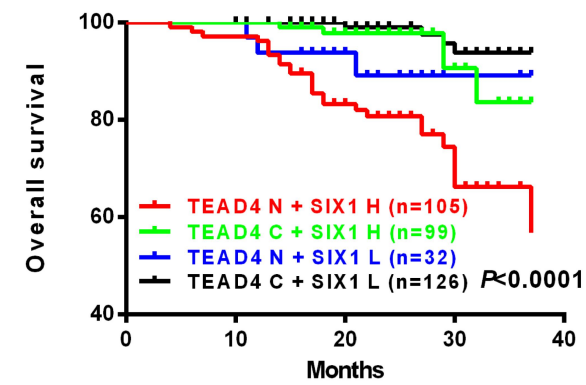

Figure 4 TEAD4 and SIXI are the prognostic markers for the colorectal cancer. (A) Correlation between TEAD4 and SIXI mRNA levels in 30 primary human CRC samples. The Spearman rank coefficient was used as a statistical measure of correlation. (B) Immunohistochemical analysis of TEAD4 and SIXI in the stage I-III colorectal cancer samples and normal epithelial tissue. Representative images of TEAD4 nuclear expression (N) (a)/SIXI high expression (H) (B), low level/cytoplasmic expression of TEAD4 (C) (c)/SIXI high expression (H) (D), TEAD4 nuclear expression (N) (e)/SIXI low expression (L) (F), low level/cytoplasmic expression of TEAD4 (C) (G) /SIXI low expression (L) (h), TEAD4 (i) and SIXI (j) in normal epithelial tissue were shown. (C) Association of TEAD4 nuclear expression and SIXI expression in 362 stage I-III CRC samples. $\chi 2$ test was used to evaluate the association. $* * * P<0.00 I$. (D) The Mann-Whitney U-test was performed to assess statistical significance of SIXI expression levels in CRC and normal epithelia. (E) Kaplan-Meier plots of overall survival of the stage I-III CRC patients stratified by SIXI expression. Log Rank test was performed to assess statistical significance. (F) Kaplan-Meier plots of overall survival of the stage I-III CRC patients stratified by nuclear TEAD4 expression. Log Rank test was performed to assess statistical significance. (G) Kaplan-Meier plots of overall survival of the stage I-III CRC patients stratified by combination of SIXI and nuclear TEAD4 expression. Log Rank test was performed to assess statistical significance. 
Table I Univariate and Multivariable Analyses for TEAD4 and SIXI in OS in Colon Cancer Patients

\begin{tabular}{|l|l|l|l|}
\hline \multirow{2}{*}{} & \multicolumn{2}{l|}{ OS } & \multicolumn{2}{l|}{ n (Events) } \\
\cline { 2 - 4 } & HR (95\% CI) & $P$ & \\
\hline Univariate & & & $257(I 2)$ \\
Others & I & & $105(26)$ \\
TEAD4(N)/SIXI(H) & $5.82(2.94-I I .54)$ & $<0.00 I$ & \\
Multivariable & & & \\
TEAD4(N)/SIXI(H) & $5.96(2.86-I 2.42)$ & $<0.00 I$ & \\
Sex & $0.74(0.39-I .40)$ & 0.350 & \\
Age & $I .32(0.69-2.50)$ & $0.40 I$ & \\
Stage & & & \\
III vs I/II & $2.25(I .13-4.47)$ & $0.02 I$ & \\
Grade & & 0.110 & \\
Mod/well dif. vs & $0.29(0.06-I .33)$ & & \\
Poorly/Undif. dif. & & \\
\hline
\end{tabular}

Note: Multivariable analysis adjusted for age, gender, AJCC stages (I-III), and grade of differentiation (well differentiated, moderately differentiated, poorly differentiated, and undifferentiated).

Abbreviations: TEAD4(N), TEAD4 nuclear expression; SIXI(H), SIXI high expression.

TEAD4 nuclear expression with SIX1 overexpression (Table 1) were the independent poor prognostic markers for the stage I-III CRC patients. Taken together, our data indicate that TEAD4 promotes EMT and cell migration through directly targeting SIX1 in colorectal cancer, thus leading a poor prognosis in colorectal cancer patients.

\section{Discussion}

Epithelial-mesenchymal transition is associated with tumor progression, metastasis, chemoresistance and tumor recurrence. ${ }^{8}$ Recently, we have shown that transcription factor TEAD4 could promote EMT in colorectal cancer through directly targeting the mesenchymal marker vimentin. ${ }^{7}$ In this study, we further explored the mechanism of TEAD4's role in epithelial-mesenchymal transition. SIX1, another transcription factor related with EMT, was identified as a new direct target gene of TEAD4 mediating the function of TEAD4 to inhibit CDH1 expression and promoting cell migration in colorectal cancer. The viability and invasive activity might be further studied and discussed in the future. Interestingly, SIX1's expression is also independent on the YAP, the well-established co-activator of TEAD4, which further demonstrates the YAP independent role and unique function of overexpressed TEAD4 in the progression of colorectal cancer. Further studies need to explore the new potential co-activator for transcription factor TEAD4, whose protein interaction could be a new therapeutic drug target.
Also, in vivo experiments might be further studied and discussed in the future.

Overexpression of SIX1 has been related to the tumorigenesis, EMT, lymphangiogenesis and metastasis. ${ }^{4}$ Gene amplification is a mechanism of SIX1 overexpression in breast cancer. ${ }^{9}$ In colorectal cancer, miR-30b has been reported to target SIX1. ${ }^{10}$ Low expression level of miR$30 \mathrm{~b}$ can result in SIX1 overexpression in colorectal cancer. Here, we show that overexpression of TEAD4 promotes transcription of SIX1, which can also lead to SIX1 overexpression in colorectal cancer. Our study provides a novel dysregulation mechanism of SIX1 overexpression in colorectal cancer. Previously, we found that overexpression and nuclear expression of TEAD4 is correlated with distant metastasis in colorectal cancer. Interestingly, we also noticed that clinicopathological correlation analysis showed that TEAD4 is associated with lymph node metastasis in colorectal cancer. ${ }^{7}$ In a mouse model of breast cancer, SIX1 can induce lymphangiogenesis and metastasis through upregulation of VEGFC. ${ }^{11}$ Thus, TEAD4 could promote lymph node metastasis by increasing SIX1 expression in colorectal cancer, which needs to be explored in the further study.

EMT-related transcription factors could be the prognostic markers for multiple cancers. ${ }^{2}$ Overexpression of SIX1 is correlated with poor prognosis in breast cancer, liver cancer ${ }^{12}$ and prostate cancer. ${ }^{13}$ Recently, SIX1 is reported as an independent prognostic marker for the stage I-III colorectal cancer patients, ${ }^{6}$ who are without metastases at first diagnosis but under high risk of tumor recurrence and metachronous metastasis after radical surgery. In our cohort, overexpression of SIX1 is correlated with poor prognosis and is also an independent prognostic marker for colorectal cancer patients. Since SIX1 is a direct target gene of TEAD4 in colorectal cancer. TEAD4 can regulate other target genes, such as vimentin, to promote tumor progression. Thus, it is not surprising that overexpression and nuclear expression of TEAD4 alone is an independent prognostic marker in our cohort. Interestingly, we found that nuclear expression TEAD4 with SIX1 overexpression is associated with the poorest overall survival in the colorectal cancer. Combination of nuclear TEAD4 and SIX1 overexpression could be a better prognostic marker for the stage I-III colorectal cancer. Altogether, our results show a new mechanism of TEAD4 regulating the epithelial-mesenchymal transition and the prognostic value of nuclear TEAD4 with 
overexpression of its new direct target gene SIX1 in colorectal cancer.

\section{Acknowledgments}

The work was supported in part by grants \#81672517, \#81570474, \#81502020, \#82073056, and \#82002507 from the National Natural Science Foundation of China, Shanghai Pujiang Program (19PJ1407600) and sponsored by Shanghai Municipal Commission of Health and Family

Planning (\#201740122).

\section{Disclosure}

The authors declare no competing interests.

\section{References}

1. Manfredi S, Lepage C, Hatem C, Coatmeur O, Faivre J, Bouvier AM. Epidemiology and management of liver metastases from colorectal cancer. Ann Surg. 2006;244(2):254-259. doi:10.1097/01. sla.0000217629.94941.cf

2. Kalluri R, Weinberg RA. The basics of epithelial-mesenchymal transition. J Clin Invest. 2009;119(6):1420-1428. doi:10.1172/ JCI39104

3. Kahlert C, Lahes S, Radhakrishnan P, et al. Overexpression of ZEB2 at the invasion front of colorectal cancer is an independent prognostic marker and regulates tumor invasion in vitro. Clinical Cancer Res. 2011;17(24):7654-7663. doi:10.1158/1078-0432.CCR-10-2816

4. Wu W, Ren Z, Li P, et al. Six1: a critical transcription factor in tumorigenesis. Int $J$ Cancer. 2015;136(6):1245-1253. doi:10.1002/ ijc. 28755
5. Ono H, Imoto I, Kozaki $\mathrm{K}$, et al. SIX1 promotes epithelial-mesenchymal transition in colorectal cancer through ZEB1 activation. Oncogene. 2012;31(47):4923-4934. doi:10.1038/ onc.2011.646

6. Kahlert C, Lerbs T, Pecqueux M, et al. Overexpression of SIX1 is an independent prognostic marker in stage I-III colorectal cancer. Int J Cancer. 2015;137(9):2104-2113. doi:10.1002/ijc.29596

7. Liu Y, Wang G, Yang Y, et al. Increased TEAD4 expression and nuclear localization in colorectal cancer promote epithelial-mesenchymal transition and metastasis in a YAP-independent manner. Oncogene. 2016;35(21):2789-2800. doi:10.1038/onc.2015.342

8. Lamouille S, Xu J, Derynck R. Molecular mechanisms of epithelial-mesenchymal transition. Nat Rev Mol Cell Biol. 2014;15 (3):178-196.

9. Reichenberger KJ, Coletta RD, Schulte AP, Varella-Garcia M, Ford HL. Gene amplification is a mechanism of Six1 overexpression in breast cancer. Cancer Res. 2005;65(7):2668-2675. doi:10.1158/ 0008-5472.CAN-04-4286

10. Zhao H, Xu Z, Qin H, Gao Z, Gao L. miR-30b regulates migration and invasion of human colorectal cancer via SIX1. Biochem J. 2014;460(1):117-125. doi:10.1042/BJ20131535

11. Wang CA, Jedlicka P, Patrick AN, et al. SIX1 induces lymphangiogenesis and metastasis via upregulation of VEGF-C in mouse models of breast cancer. J Clin Invest. 2012;122(5):1895-1906. doi:10.1172/ JCI59858

12. Ng KT, Man K, Sun CK, et al. Clinicopathological significance of homeoprotein Six1 in hepatocellular carcinoma. $\mathrm{Br} J$ Cancer. 2006;95(8):1050-1055. doi:10.1038/sj.bjc.6603399

13. Zeng J, Shi R, Cai CX, et al. Increased expression of Six 1 correlates with progression and prognosis of prostate cancer. Cancer Cell Int. 2015;15:63. doi:10.1186/s12935-015-0215-Z

\section{Publish your work in this journal}

Cancer Management and Research is an international, peer-reviewed open access journal focusing on cancer research and the optimal use of preventative and integrated treatment interventions to achieve improved outcomes, enhanced survival and quality of life for the cancer patient.
The manuscript management system is completely online and includes a very quick and fair peer-review system, which is all easy to use. Visit http://www.dovepress.com/testimonials.php to read real quotes from published authors. 